\title{
Und sie bewegt sich doch!
}

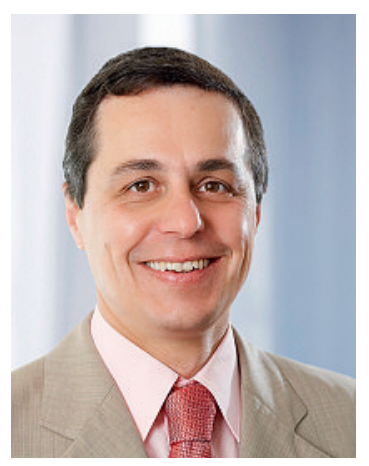

Kaum zu glauben. Auf Hochtouren laufen nicht nur die Umsetzung bereits entschiedener Gesundheitsreformen (Spital- und Pflegefinanzierung, Risikoausgleich 1) und die Neuordnung der ambulanten Medizin (integrierte Versorgung, Ende des Zulassungsstopps, Risikoausgleich 2) ... nein, eine neue Reform steht an: die Präventionsreform.

Der 25. März 2010 war ein wichtiger Tag für die Geschichte der Prävention in der Schweiz: die Gesundheitskommission des Nationalrates hat nach fünf Stunden Hearing und Debatte - die FMH war dabei - mit 13 zu 7 Stimmen «Eintreten» entschieden. Im politischen Jargon heisst es, die Kommission will sich mit einem neuen Präventionsgesetz auseinandersetzen. Der Bundesrat hatte dem Parlament Ende September 2009 einen Gesetzesvorschlag unterbreitet. Ein neues Präventionsgesetz könnte somit entstehen, mehr als 25 Jahre nach dem Scheitern des letzten Versuches. «Könnte» - weil noch viele Hürden zu überwinden sind. Die Kommission kann nämlich am Ende ihrer Beratung in der Schlussabstimmung noch «Nein» sagen. Sagt sie hingegen «Ja», dann muss der Nationalrat ihr auch folgen. Dann ist erst die erste Kammer einverstanden, die zweite - der Ständerat kommt noch. Der Weg hat also soeben begonnen.
Verhütung übertragbarer Krankheiten (Impfungen). Stets hat man darauf geachtet, dass sich unterschiedliche Massnahmen optimal ergänzen: Erziehung in der Schule und Information für die Bevölkerung, verbunden mit sinnvollen Bestimmungen, wie etwa die Gurtentragpflicht oder die 0,5-PromilleLimite. Die Kostenwirksamkeit von Präventionsmassnahmen wurde neulich auch für die Schweiz belegt: so etwa in der 2009 veröffentlichten INFRAS-Studie der VIPS (Vereinigung Pharmafirmen in der Schweiz) oder in der BAG-Studie von 2010.

\section{Das Präventionsgesetz führt zu keinen Mehrkosten, sondern zur Effizienzsteigerung}

Das neue Präventionsgesetz wird dafür sorgen, dass Doppelspurigkeiten zwischen Bund, Kantonen, Gesundheitsligen und den Gesundheitsakteuren verschwinden. Mit gemeinsam erarbeiteten nationalen Zielen ist die Grundlage für eine kohärente Strategie und einen effizienten Mitteleinsatz gelegt. Die Kantone bleiben die Drehscheibe der Prävention, und die nationalen Strukturen werden vereinfacht: Anstelle von drei verschiedenen Betrieben mit je einer Verwaltung, einer Buchhaltung, einer Internetseite usw. (Gesundheitsförderung Schweiz, Tabakpräventionsfonds und die Abteilung

\section{Das Präventionsgesetz wird dafür sorgen, dass Doppelspurigkeiten zwischen Bund, Kantonen, Gesundheitsligen und den Gesundheitsakteuren verschwinden}

Dennoch bin ich zuversichtlich: Auch die Schweiz ist sich heute der Bedeutung von Prävention und Gesundheitsförderung bewusst. Das neue Gesetz ist ein Organisationsgesetz, das die Steuerung und die Koordination im Bereich Primärprävention und Gesundheitsförderung verbessern will. Die Sekundärprävention hat mit diesem Gesetz nur dann zu tun, wenn sie kollektiv angelegt ist (Screening-Programme). Die individuelle Sekundärprävention ist wie bis anhin im KVG geregelt. Mit Hilfe dieses Gesetzes können wir bewährte regionale Strategien national weiterentwickeln und so auch einen wichtigen Beitrag zur Lebensqualität vieler Menschen leisten. Zu Mehrkosten führt das Gesetz nicht, sondern zur Effizienzsteigerung. Über konkrete Präventionsprogramme steht im Gesetz nichts: Es wird gemeinsame Aufgabe von Bund, Kantonen und Drittorganisationen sein, konkrete Präventionsziele zu definieren und entsprechende Programme zu finanzieren.

Bewährt hat sich die Prävention in den letzten Jahrzehnten in vielen Bereichen: von der Zahngesundheit über die Verhinderung von Verkehrsunfällen bis zum Lungenkrebs (Passivrauchgesetz), Brustkrebs (Screening-Programme) und
Präventionsprogramme des BAG) ist nur noch eine Organisation tätig, mit entsprechenden Effizienzgewinnen. Über dieses Umsetzungsorgan wird man aber noch viel reden: Das vom Bundesrat vorgeschlagene nationale Institut hat wenig Chancen, das Licht der Welt zu erblicken.

Die Wirtschaftsverbände sind skeptisch. Sie befürchten Mehrkosten für die Arbeitsgeber, eine Einschränkung der Handelsfreiheit gewisser Branchen (Alkohol, Tabak, Werbung usw.) sowie die Einengung der Bevölkerung, sich frei - d.h. auch gesundheitsschädlich - zu verhalten. Doch beim neuen Präventionsgesetz geht es um eine bessere und kostenneutrale Organisation der Prävention und keineswegs um eine Einschränkung der Rechte des Bürgers.

Fazit: Im Bereich der Prävention liegt ein Potential, dessen Nutzung für die Schweiz unverzichtbar ist, wenn unser Gesundheitswesen effizient und bezahlbar bleiben soll.

Das hat inzwischen auch eine Mehrheit der Politiker gemerkt. Heureka - und sie bewegt sich doch ... die Gesundheitspolitik!

Dr. med. Ignazio Cassis, FMH-Vizepräsident und Nationalrat 